Fortes-Vilaltella, M., Oriol, X., Filella, G., Del Arco, I., \& Soldevila, A. (2013). Inteligencia emocional y personalidad en las diferentes áreas de conocimiento de los estudiantes universitarios. Revista Electrónica Interuniversitaria de Formación del Profesorado, 16(1), 109-120.

DOI: http://dx.doi.org/10.6018/reifop.16.1.179471

\title{
Inteligencia emocional y personalidad en las diferentes áreas de conocimiento de los estudiantes universitarios
}

\author{
Marta Fortes-Vilaltella, Xavier Oriol, Gemma Filella, Isabel del Arco, Anna Soldevila \\ Universidad de Lleida
}

\section{Resumen}

El motivo de este estudio fue poner de manifiesto el estado de la competencia socioemocional, considerada competencia transversal en el nuevo marco universitario, y su relación con personalidad. Se analizó la competencia emocional (EQ-i) y la personalidad (NEO-FFi) de 640 alumnos de $1^{\text {er }}$ curso de diferentes áreas de conocimiento de la UdL (231 hombres, 409 mujeres, $M=18.7$ y DT $=1.9)$.

Se realizaron pruebas Turkey, correlaciones entre los resultados y un análisis de regresión múltiple para determinar si existían diferencias entre los resultados por titulaciones y sexo. Se determinaron esas diferencias y también correlaciones entre competencia emocional y personalidad.

\section{Palabras clave}

Inteligencia emocional; personalidad; formación universitaria.

\section{Emotional intelligence and personality in the different areas of knowledge of university students}

\section{Abstract}

The aim of this study was to show the state of the emotional competence, considered transferable competence in the new university framework, and its relation with personality. We analyzed the emotional competition (EQ-i) and the personality (NEO-FFi) of 640 students of 1st year of different areas of knowledge at the UdL (231 men, 409 women, $M=18.7$ and SD =1.9). 
Tests Turkey, interrelations between the results and a multiple regression analysis were realized to determine if there exist differences between the areas of knowledge and gender. Those differences were identified and also correlations between emotional competence and personality.

\section{Key words}

Emotional intelligence; personality; university training.

\section{Introducción}

La pretensión de los países que integran la comunidad europea de convertirse en una sociedad basada en el conocimiento, más competitiva y dinámica se ha plasmado en la formulación de unos objetivos educativos comunes. Por eso estamos asistiendo a profundos cambios en el sistema educativo en todos sus niveles de formación (LOE, 2006), todos orientados al desarrollo integral centrado en competencias, en la calidad educativa y en dar respuesta a las demandas socioprofesionales actuales. El proceso de cambio en el que se encuentra el sistema universitario plantea nuevas oportunidades para mejorar la oferta formativa y sus resultados (Palomera, FernándezBerrocal y Brackett, 2008).

Los nuevos planes de estudio incluyen algunas competencias que permiten mejorar la inserción sociolaboral de los estudiantes universitarios. En este sentido, las competencias sociopersonales aparecen como algunas de las más demandadas en las prácticas profesionales en los estudiantes universitarios y las que están adquiriendo una mayor relevancia en el ámbito profesional (Goleman, Boyatzis y McKee, 2002; Alberici y Serreri, 2005; Durán-Aponte y Durán-García, 2012). Estos datos coinciden con los hallados en los estudios científicos relacionados con el desarrollo de las competencias emocionales en la práctica profesional y los beneficios que estas aportan. Diferentes estudios señalan que la inteligencia emocional (IE) mejora la interacción de los empleados con sus compañeros, las estrategias que utilizan para manejar los conflicto y el estrés, y el rendimiento general en el trabajo (Ashkanasy y Daus, 2005). Los trabajadores que obtienen mayores puntuaciones en inteligencia emocional también son percibidos por sus compañeros y sus superiores como personas que facilitan las relaciones interpersonales, toleran el estrés y con un gran potencial de liderazgo (Lopes, Cote, y Salovey, 2006).

Otro de los aspectos muy relevantes a tener en cuenta en el desarrollo de las competencias en el marco del sistema europeo es observar el grado de influencia que el desarrollo de este tipo de competencias puede ejercer en la motivación hacia el aprendizaje y el rendimiento académico. De acuerdo con los estudios neurocientíficos, los procesos emocionales tienen un profundo efecto en los elementos de la cognición tales como: aprendizaje, atención, memoria, toma de decisiones y relaciones sociales (Immordino-Yang y Damasio 2006; Damasio, 2010). Ello ha sido corroborado por los estudios destinados a la evaluación de programas de educación emocional en la escuela. Se ha observado que el desarrollo de este tipo de competencias en los niños predice la motivación para el aprendizaje, la adaptación al entorno escolar, la participación en los centros y las buenas relaciones con los compañeros y profesores (Durlak, Weissberg, Dymnicki, Taylor y Shellinger, 2011; Duncan et al., 2007).

A pesar de todo lo expuesto hasta el momento, los estudios sobre el desarrollo de competencias no han estado exentos de críticas y muchos autores han destacado que las competencias son rasgos de la personalidad como por ejemplo el control de impulsos o la responsabilidad social (Neubauer y Freudenthaler 2005). Goleman, (1995) que contempla constructos como la empatía y la asertividad que pueden ser consideradas también del dominio de la personalidad. Aspecto que choca frontalmente con los modelos que consideran que cualquier competencia es aprendida o susceptible de aprendizaje y desarrollo (Repetto, Ballesteros y Malik, 2000; Bisquerra, 2002; Roe, 2002). Esta misma discusión se ha producido en la investigación relativa a la consideración de la inteligencia emocional como constructo. Diversos autores contemplan un modelo basado en las competencias mentales de la IE que parte de la idea de que el entrenamiento puede producir efectos y por lo tanto la IE puede ser aprendida (Mayer, J. D., Salovey, P., y Caruso, D. R. 2000). Otros autores consideran la 
inteligencia emocional como habilidad o rasgo de personalidad (Petrides y Furnham, 2003). Por su parte, los modelos mixtos combinan dimensiones de personalidad como el optimismo y la capacidad de automotivación con habilidades emocionales (Goleman, 1995; Bar-On, 1997; Goleman, 1998).

Todos los estudios revisados ponen de manifiesto dos aspectos muy relevantes a tener en cuenta y que creemos muy interesantes a explorar. Por un lado y de acuerdo con el nuevo plan de estudios adaptado a la EEES, resulta interesante poder observar las diferencias existentes en los alumnos que acceden a la universidad respecto a las competencias emocionales dependiendo del ámbito del área de conocimiento. Ello nos permitirá obtener información relevante de cara a la planificación de estos estudios, teniendo en cuenta que las competencias emocionales son algunas de las más demandas durante las prácticas profesionales (Durán-Aponte y Durán-García, 2012). En segundo lugar, no existen demasiados estudios que analicen las relaciones de la IE con determinados rasgos de personalidad. Por ello, también se ha considerado interesante observar si existen diferencias en los rasgos de personalidad entre los alumnos que acceden a la universidad, teniendo en cuenta el área de conocimiento y además analizar las relaciones existentes entre los rasgos de personalidad (neuroticismo, extraversión, apertura, amabilidad y responsabilidad) y la IE y comprobar el grado de predicción de los rasgos respecto a los factores de la IE.

En esta investigación 1) se esperan hallar mayores puntuaciones en los factores de IE en los estudiantes de las áreas de conocimiento de letras y ciencias sociales; 2) se prevén mayores puntuaciones en extraversión y apertura en los estudiantes de las áreas de letras y ciencias sociales; 3) se esperan hallar relaciones positivas y negativas entre factores de la IE y rasgos de personalidad; 4) se espera que algunos rasgos de personalidad actúen como variables predictoras de la IE.

\section{Metodología}

\section{Participantes}

La muestra definitiva estuvo compuesta por $N=640$ estudiantes ( 231 hombres y 409 mujeres) de $1 \mathrm{r}$ curso de la Universidad de Lleida $\left(M_{\text {edad }}=18,7\right.$; $\left.D T=1,9\right)$. La mortalidad de la muestra fue del $2,9 \%$ lo que ocasionó una pérdida de 19 sujetos en el estudio.

Para la selección de los participantes se incluyeron titulaciones de cada una de las áreas de conocimiento por las que se distribuyen los estudios en la Universidad de Lleida. Del área de letras se incluyeron las titulaciones de: Historia, Historia del Arte, Geografía y Ordenación del Territorio, Letras y Estudios Hispánicos: Lengua y Literatura. Del área correspondiente a ciencias de la salud se seleccionaron las siguientes titulaciones: Ciencias y salud animal e Enfermería. Del área de ciencias sociales se seleccionaron las titulaciones de: Administración y dirección de empresas, Educación social, Magisterio de educación primaria y Psicología. En los estudios de Ingeniería se encuentran las titulaciones de Ingeniería Industrial e Ingeniería de la Edificación.

\section{Instrumentos}

Para llevar a cabo la investigación se utilizaron dos pruebas:

a) El Inventario de Cociente Emocional (EQ-i) (Bar-On, 1997), en la versión traducida y validada al español por Nelly Ugarriza Chávez (2001) constituye una medida de autoinforme de la IE. Incluye 133 afirmaciones y está compuesto por cinco factores principales que se descomponen en 15 subescalas o habilidades relacionadas con dichos factores: a) Inteligencia intrapersonal, que recoge las habilidades de autoconocimiento emocional, asertividad, autoconsideración, autoactualización e independencia; b) Inteligencia interpersonal, que comprende las subescalas de empatía, responsabilidad social y relaciones interpersonales; c) Adaptabilidad, compuesta por las habilidades de solución de problemas, comprobación de la realidad y flexibilidad; d) Manejo del estrés, que evalúa las subescalas de tolerancia al estrés y control de impulsos; y e) Estado de ánimo, integrada por las habilidades de felicidad y optimismo. Además de evaluar estas 15 subescalas, el inventario incluye cuatro indicadores de validez que miden el grado de distorsión 
de las respuestas de los sujetos, a fin de reducir el efecto de deseabilidad social e incrementar la seguridad de los resultados obtenidos.

El EQ-i abarca varias competencias emocionales y sociales, proporcionando no sólo la estimación del nivel de IE sino también un perfil social y afectivo. Cada ítem expresa un determinado estado emocional y el sujeto debe mostrar su grado de acuerdo con cada uno de ellos en una escala tipo Likert de cinco puntos (1=De acuerdo; 5=Desacuerdo).

b) El Inventario de Personalidad NEO-FFI es la versión reducida del NEO-PI-R, uno de los instrumentos más utilizados en la evaluación de los Cinco Grandes, elementos básicos de la estructura personal obtenidos a partir de la hipótesis léxica en el estudio de la personalidad. Hemos utilizado la adaptación española realizada por la editorial TEA sobre 2.000 personas, que ha demostrado unos índices de fiabilidad y validez adecuados27: coeficientes alfa de Cronbach entre 0,82 y 0,90; estructura pentafactorial idéntica al inventario original. Consta de 60 ítems que se puntúan según una escala tipo Likert de cinco puntos. Brevemente, las cinco puntuaciones ofrecidas por el inventario son Neuroticismo (nivel de inestabilidad emocional), Extraversión (nivel de energía y sociabilidad), Apertura (nivel de curiosidad intelectual y sensibilidad estética), Amabilidad (nivel de las tendencias interpersonales de acercamiento o rechazo de los demás) y Responsabilidad (nivel de autocontrol y autodeterminación).

\section{Procedimiento}

Se administraron los 2 cuestionarios a 640 alumnos de las titulaciones citadas en la muestra. Para la selección de las titulaciones, partimos de un tipo de muestreo no probabilístico, por accesibilidad, teniendo en cuenta siempre que la muestra fuese representativa en la población limitada en las diversas facultades y del total de alumnos de primer curso de la Universidad de Lleida.

Respetando la ley de protección de datos se creó un sistema de identificación personal anónimo para los estudiantes que participaron en el estudio y se pidió el permiso correspondiente a los coordinadores de titulación para el acceso a la muestra.

Un investigador del grupo informó a los alumnos de cada titulación sobre los objetivos de la investigación y les acompañó en el proceso de cumplimentación de los mismos. Los cuestionarios fueron administrados online.

\section{Resultados}

Para el análisis de resultados se utilizó el paquete estadístico SPSS 18.0. En primer lugar se realizaron comparaciones por sexos mediante pruebas $\mathrm{T}$ para observar si existían diferencias tanto en las competencias emocionales como en los cinco factores de personalidad. A posteriori se realizó una correlación entre el cuestionario EQ-i y el cuestionario de personalidad NEO-FFI y para finalizar se realizó un análisis de regresión múltiple por pasos sucesivos.

\section{Diferencias por sexos en el análisis de las competencias emocionales (EQ-i)}

Se realizaron comparaciones mediante pruebas T en las cinco dimensiones del EQ-i, incorporando también el índice global de inteligencia emocional. Los resultados de las mostraron diferencias por sexos en 2 dimensiones. En concreto, las chicas obtuvieron puntuaciones superiores en la dimensión correspondiente a las habilidades interpersonales $(t=-7.707, g l=564, p<.001)$ y los chicos obtuvieron puntuaciones superiores en las habilidades de manejo del estrés $(t=3.180, g l=564, p=$ .02). (Ver tabla 1.)

\section{Diferencias por sexos en el análisis de los cinco factores del NEO-FFI}

Se realizaron también comparaciones por sexos mediante pruebas $\mathrm{T}$ de los cinco factores de personalidad del NEO-FFI. Los resultados de las pruebas mostraron que existen diferencias entre sexos en tres de los cinco factores de personalidad. En concreto, las chicas puntuaron más alto en 
Amabilidad ( $t=-4.597, \mathrm{gl}=638, p<.001)$; en Responsabilidad $(t=-2.773, \mathrm{gl}=638, p=.06)$ y en Neuroticismo $(t=-6.775, g l=638, p<.001)($ ver tabla 1$)$.

Tabla 1. Diferencias por sexos en el análisis de los factores del EQ-i y NEO-FFI Estadísticos descriptivos correspondiente a las diferencias entre sexos en los componentes del EQ-i

\begin{tabular}{llcl}
\hline Componentes y factores & Muestra & Media & D.T \\
\hline Inteligencia Emocional & Chicos & 99.8 & 13.7 \\
Global & Chicas & 111.5 & 15.9 \\
C. Intrapersonales & Chicos & 102.4 & 13. \\
& Chicas & 107.1 & 15.1 \\
C. Interpersonales & Chicos & 101.1 & 16. \\
& Chicas & 106.8 & 13.9 \\
C. Adaptabilidad & Chicos & $95.8^{*}$ & 10.6 \\
C. Ánimo general & Chicas & & \\
Manejo del estrés & Chicos & $99.6^{*}$ & 16.5 \\
& Chicas & & \\
& Chicos & &
\end{tabular}

Estadísticos descriptivos correspondiente a las diferencias entre sexos en los componentes del NEO-FFI

\begin{tabular}{llcl}
\hline Componentes y factores & Muestra & Media & D.T \\
\hline Neuroticismo & Chicos & 99.8 & 13.7 \\
& Chicas & 111.5 & 15.9 \\
Extraversión & Chicos & 102.4 & 13. \\
& Chicas & 107.1 & 15.1 \\
Apertura a la experiencia & Chicos & 101.1 & 16. \\
& Chicas & 106.8 & 13.9 \\
Amabilidad & Chicos & $95.8 *$ & 10.6 \\
& Chicas & & \\
Responsabilidad & Chicos & & \\
& Chicas & $99.6 *$ & 16.5 \\
* $0<.05$ & & &
\end{tabular}

\section{Diferencias por titulaciones en el análisis de las habilidades emocionales (EQ-i)}

Para el análisis entre las diferentes titulaciones, se agruparon los diferentes tipos de estudios en cada una de las áreas temáticas a las que pertenecen. Se diferenció entre las especialidades de: Letras, Ingenierías, Ciencias sociales y Ciencias de la salud. El análisis de los resultados se realizó mediante ANOVAS entre todos los grupos con las posteriores pruebas post-hoc de Tukey para realizar comparaciones dos a dos.

El análisis de varianza mostró diferencias entre todos los grupos en las dimensiones de inteligencia emocional global $(F=2,819 ; g l=3.520 ; p=.030)$; en la dimensión de habilidades interpersonales $(F=$ 
3.459; $g l=3.520 ; p=.010)$, manejo del estrés $(F=3.459 ; g l=3.520 ; p=.002)$ y el estado de ánimo general $(F=2.969 ; g l=3.520 ; p=.030)$.

Las posteriores comparaciones con la prueba de Tukey del EQ-i mostraron diferencias en las habilidades interpersonales entre las titulaciones de ciencias sociales y las de ingeniería a favor de las primeras $(p=.040)$ y en las habilidades de manejo del estrés entre las titulaciones de letras y ciencias sociales, en concreto las titulaciones de letras obtuvieron mayores puntuaciones $(p=.002)$. En las habilidades del estado de ánimo general los estudiantes de ciencias sociales obtuvieron mayores puntuaciones que los estudiantes de ciencias de la salud $(p=.020)$.

\section{Diferencias por titulaciones en el análisis de los cinco factores del NEO-FFI}

Para el análisis de resultados de nuevo se realizó un ANOVA entre todos los grupos y a posteriori pruebas post-hoc de Tukey.

Los resultados del análisis de varianza mostraron diferencias entre los grupos en el factor de extraversión $(F=5.312 ; g l=3.586 ; p=.001)$; en el factor de apertura a la experiencia $(F=7.202 ; g l=$ $3.586 ; p<.001)$ y de neuroticismo $(F=4.132 ; g l=3.586 ; p=.006)$.

Las posteriores comparaciones con la prueba de Tukey mostraron diferencias en el componente de extraversión entre los alumnos de ciencias sociales y ciencias de la salud a favor de los primeros ( $p=$ .001). En el componente de apertura a la experiencia entre los estudiantes de las titulaciones de ciencias sociales y de ingenierías a favor de los primeros $(p<.001)$ y en neuroticismo, donde los estudiantes de ciencias de la salud obtuvieron puntuaciones superiores a los estudiantes de ciencias sociales $(p=.020)($ ver tabla 2$)$

Tabla 2. Diferencias por titulaciones en el análisis de los cinco factores del EQ-I NEO-FFI Estadísticos descriptivos correspondiente a las diferencias entre titulaciones del EQ-i

\begin{tabular}{|c|c|c|c|c|}
\hline & Letras & Ciencias sociales & Ciencias de la salud & Ingeniería \\
\hline IE Global & $457.74 \pm 16.43$ & $443.14 \pm 13.14$ & $420.66 \pm 15.12$ & $438.15 \pm 10.13$ \\
\hline Intrapersonal & $153.25 \pm 13.23$ & $151.14 \pm 12.45$ & $143.33 \pm 10.24$ & $148.83 \pm 15.78$ \\
\hline Interpersonal & $99.09 \pm 7.89$ & $98.80 \pm 10.24$ & $92.86 \pm 10.69$ & $96.26 \pm 11.20$ \\
\hline Adaptabilidad & $97.00 \pm 12.05$ & $92.25 \pm 11.88$ & $90.53 \pm 9.87$ & $91.75 \pm 10.47$ \\
\hline Animo General & $69.12 \pm 11.14$ & $69.07 \pm 8.74$ & $62.20 \pm 11.59$ & $67.99 \pm 9.06$ \\
\hline Manejo del estrés & $69.16 \pm 11.20$ & $61.79 \pm 10.42$ & $58.60 \pm 10.72$ & $62.53 \pm 10.61$ \\
\hline
\end{tabular}

Estadísticos descriptivos correspondiente a las diferencias entre titulaciones en los factores del NEO-FFI

Letras Ciencias sociales
Ingeniería
$13.52 \pm 6.34$
$25.09 \pm 4.75$
$22.77 \pm 5.02$
$31.47 \pm 4.36$
$27.05 \pm 6.01$ 


\section{Relación entre las habilidades emocionales y los factores de personalidad}

Se realizó una correlación entre el cuestionario EQ-i y el cuestionario de personalidad NEO-FFI para observar las relaciones existentes entre las habilidades emocionales y los factores de personalidad. Para ello se realizó una correlación de Pearson. Los resultados mostraron correlaciones positivas entre los factores de extraversión y los componentes de la Inteligencia emocional global $(r p=.354, p$ <.001); las habilidades intrapersonales $(r p=.393, p<.001)$; las habilidades interpersonales $(r p=.449$, $p<.001)$; los componentes de adaptabilidad ( $r p=.100, p=.010)$ y los componentes del estado de ánimo general $(r p=.588, p<.001)$.

También se observaron correlaciones positivas entre apertura a la experiencia y habilidades interpersonales $(r p=.165, p<.001)$ y componentes del estado de ánimo general $(r p=.100, p=.010)$. El factor de amabilidad obtuvo correlaciones positivas con los componentes de Inteligencia emocional global ( $r p=.445, p<.001)$; las habilidades intrapersonales ( $r p=.234, p<.001$ ); los componentes interpersonales $(r p=.556, p=0.00)$; los componentes de amabilidad ( $r p=.360, p<$ .001); las habilidades de manejo del estrés ( $r p=.384, p<.001)$ y los componentes del estado de ánimo general ( $r p=.303, p<.001$ ) (ver tabla 3) El factor de neuroticismo obtuvo correlaciones negativas con Inteligencia emocional global ( $r p=-.638, p<.001)$; habilidades intrapersonales $(r p=$ $.600, p<.001)$; componentes interpersonales $(r p=-0.207, p<.001)$; habilidades de manejo de adaptabilidad ( $r p=-.491, p<.001)$; manejo del estrés $(r p=-0.629, p<.001)$ y los componentes del estado de ánimo general $(r p=-.584, p<.001)$.

Tabla 3. Correlaciones entre los factores de personalidad y los componentes del EQ-I

IE Global Intrapersonal Interpersonal Adaptabilidad E. Ánimo General Manejo del estrés

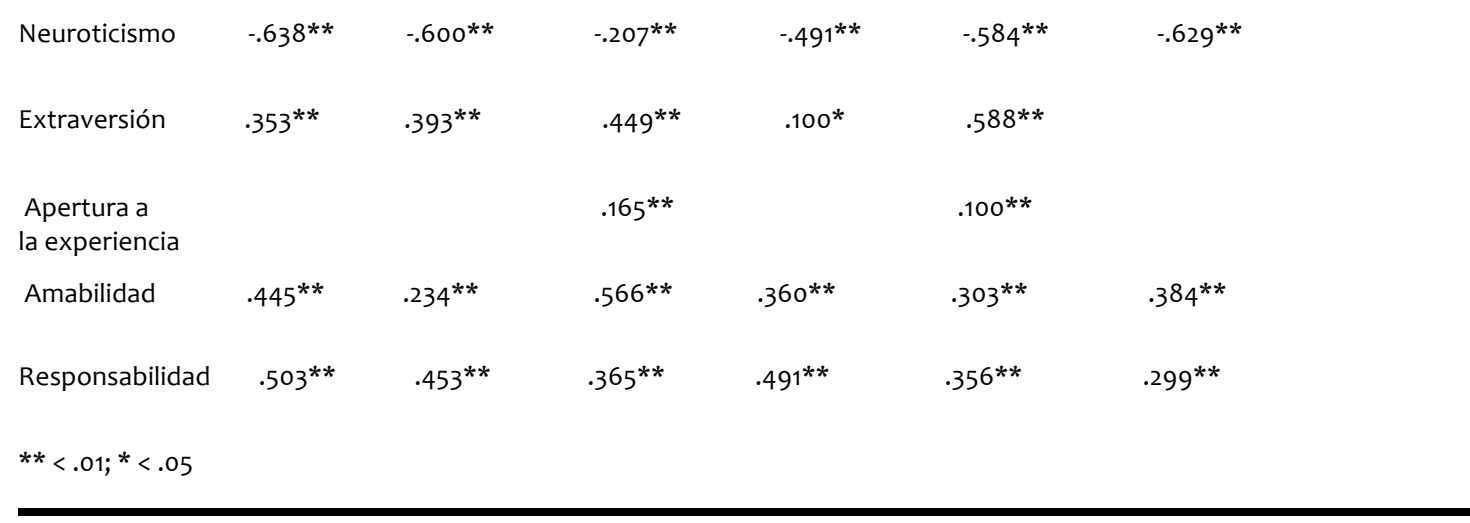

\section{Los rasgos de personalidad como predictores de la IE}

Finalmente, se realizó un análisis de regresión múltiple por pasos sucesivos (ver tabla 4). Se consideraron como variables predictoras los factores de personalidad del NEO-FFI y como variable dependiente el índice global de IE. Siguiendo el método de pasos sucesivos se ha obtenido un coeficiente de determinación igual a .63 $(F(3.365)=154.4, p<.001)$.

El neuroticismo es la variable predictora con mayor importancia relativa en la ecuación, ( $\beta$-.50; $\mathrm{p}<.000)$ seguida de la responsabilidad $(\beta$.31; $\mathrm{p}<.000)$, La adaptabilidad $(\beta .20 ; \mathrm{p}<.000)$, La extraversión $(\beta .17 ; p<.000)$ y la apertura $(\beta .07 ; p<.008)$ 
Tabla 4. Análisis de Regresión de los factores del NEO-FFI sobre las escalas del EQI

\begin{tabular}{|c|c|c|c|c|}
\hline ÍNDICE DE IE & V. Predictoras & Beta & $\mathrm{t}$ & Sig. \\
\hline Bloque 1 & Neuroticismo & -.63 & -19.69 & .000 \\
\hline \multirow[t]{2}{*}{ Bloque 2} & Neuroticismo & -.55 & -19.02 & .000 \\
\hline & Responsabilidad & .38 & 13.05 & .000 \\
\hline \multirow[t]{3}{*}{ Bloque 3} & Neuroticismo & -.51 & -18.42 & .000 \\
\hline & Responsabilidad & .32 & 11.31 & .000 \\
\hline & Adaptabilidad & .23 & 8.37 & .000 \\
\hline \multirow[t]{4}{*}{ Bloque 4} & Neuroticismo & -.49 & -18.18 & .000 \\
\hline & Responsabilidad & .30 & 11.24 & .000 \\
\hline & Adaptabilidad & .21 & 7.70 & .000 \\
\hline & Extraversión & .18 & 6.96 & .000 \\
\hline \multirow[t]{5}{*}{ Bloque 5} & Neuroticismo & -.50 & -18.47 & .000 \\
\hline & Responsabilidad & .31 & 11.42 & .000 \\
\hline & Adaptabilidad & .20 & 7.54 & .000 \\
\hline & Extraversión & .17 & 6.46 & .000 \\
\hline & Apertura & .07 & 2.68 & .008 \\
\hline \multicolumn{5}{|c|}{${ }^{*} \mathrm{p}<.05 ; * * \mathrm{p}<.01$} \\
\hline
\end{tabular}

\section{Discusión y conclusiones}

Este estudio se propuso estudiar diversos aspectos de la inteligencia emocional y de la personalidad entre alumnas y alumnos universitarios de diferentes áreas de conocimiento. Los resultados globales obtenidos ponen de manifiesto que existen diferencias entre los estudiantes universitarios pertenecientes a diversos ámbitos científico-profesionales en las puntuaciones obtenidas en diversos aspectos de la inteligencia emocional y la personalidad del mismo modo que también existe diferencias entre sexos.

En la primera hipótesis, nos planteamos hallar mayores puntuaciones en los factores de IE en los estudiantes de las áreas de conocimiento de letras y ciencias sociales que en los de las otras áreas. Los resultados muestran que los estudiantes de ciencias sociales, obtienen mayores puntuaciones en las habilidades interpersonales respecto a los estudiantes de ingenierías. En contra de lo esperado, los estudiantes de letras obtienen mayores puntuaciones en los componentes de manejo del estrés respecto a los estudiantes de ciencias sociales. Y también obtienen mayores puntuaciones en el estado de ánimo general respecto a los de ciencias de la salud. En estudios anteriores como en Sala (2002) y Castejón, Cantero y Nélida (2008) se obtuvieron resultados parecidos, donde los estudiantes de ciencias sociales obtenían resultados mayores en las habilidades interpersonales.

En la segunda hipótesis, nos planteamos que las áreas de letras y ciencias sociales obtendrían mayores puntuaciones en los rasgos de personalidad de extraversión y apertura. Los alumnos de ciencias sociales obtuvieron mayores puntuaciones en extraversión respecto a los de ciencias de la salud. En el componente apertura también se obtuvieron resultados más elevados por parte de los estudiantes de las titulaciones de ciencias sociales por encima de las ingenierías. También se encontraron diferencias en las puntuaciones en neuroticismo, donde los estudiantes de ciencias de la salud obtuvieron puntuaciones superiores a los estudiantes de ciencias sociales

En la comparación por sexos de los resultados referentes al EQ-i, se observan puntuaciones superiores por parte de las chicas en las habilidades interpersonales y puntuaciones superiores por parte de los chicos en los componentes de manejo del estrés. Estos resultados concuerdan con los obtenidos por Sala (2002), también apuntan en la misma dirección que Bar-On (1997) cuando afirmaba que los hombres tenían mayores puntuaciones en las habilidades de manejo del estrés (tolerancia al estrés y el control de los impulsos) y en la independencia mientras que las mujeres 
tenían mayor puntuación en empatía y responsabilidad social (subcomponentes de las habilidades interpersonales) y en neuroticismo.

En la tercera hipótesis, se esperaba hallar relaciones positivas y negativas entre factores de la IE y rasgos de personalidad. Los resultados obtenidos muestran relaciones negativas entre neuroticismo y las dimensiones que evalúa el cuestionario de inteligencia emocional EQiS; intrapersonal, interpersonal, manejo del estrés, adaptabilidad, humor general y el índice global. También se observan relaciones positivas entre los factores de extraversión y el índice global de Inteligencia emocional, las habilidades intrapersonales, las habilidades interpersonales, los componentes de adaptabilidad y los componentes del estado de ánimo general. Y relaciones positivas también entre apertura a la experiencia y las habilidades interpersonales y componentes del estado de ánimo general. El factor de amabilidad obtuvo relaciones positivas con el índice global de inteligencia emocional, las habilidades intrapersonales, las habilidades interpersonales, las habilidades de manejo del estrés y los componentes del estado de ánimo general.

De los resultados, se desprende de forma consistente que las medidas de autoinforme de la inteligencia emocional presentan correlaciones moderadas y, a veces incluso altas, con variables de personalidad consolidadas como los cinco grandes (Austin, Saklofske y Egan, 2005; Davies, Stankov y Roberts, 1998; Dawda y Hart, 2000).

En relación a la última hipótesis referente a la predicitibilidad del índice global de IE por los rasgos de personalidad, los resultados mostraron que el neuroticismo es el rasgo que mejor predice negativamente el índice global de IE. En menor medida se encontraron la adaptabilidad, la extraversión y la apertura como rasgos predictores de IE. Baños y Pérez (2005) destacan la importancia de las competencias genéricas o transversales de tipo socioemocional en la formación del graduado universitario. Esto se corrobora cuando se obtiene que una de las competencias transversales más valoradas en el proyecto Tunning de formación universitaria es la inteligencia interpersonal (González y Wagenaar, 2003). La formación de un profesional y la apuesta por su empleabilidad en los tiempos que corren exige tanto el desarrollo de su competencia técnica como de sus competencias socioemocionales, especialmente cuando en el ejercicio de su profesión sea habitual el trabajo en equipo, la coordinación con otros profesionales, y/o la atención o trato directo con clientes. (Repetto, E., Pérez-Gonzalez, J.C. 2007). Las competencias socioemocionales son importantes para el desarrollo profesional, así como presumiblemente también para la inserción laboral y la empleabilidad (Palací y Topa, 2002; Palací y Moriano, 2003). Cherniss (2000) apunta de manera más global que las competencias socioemocionales son críticas para el desempeño efectivo en la mayoría de los trabajos.

Numerosos estudios demuestran que las competencias emocionales se relacionan con niveles óptimos de desempeño en ámbito personal, educativo, profesional y en el afrontamiento exitosos de los retos en la vida diaria. Por ello creemos necesario incorporar el desarrollo de las competencias socio-emocionales en el contexto de la enseñanza superior universitaria, El EEES nos brinda una buena oportunidad para introducir dichas competencias en el currículum de las diferentes carreras universitarias de grado (Pérez, Filella, Soldevila, 2010). En este sentido, los resultados de este estudio enfatizan la importancia de incorporar dichas competencias en los planes de estudios de los

La enseñanza de estas habilidades depende de forma prioritaria de la práctica, el entrenamiento y su perfeccionamiento, y no tanto de la instrucción verbal. Fernández-Berrocal y Ruiz (2008) afirman que lo esencial es ejercitar y practicar las habilidades emocionales y convertirlas en una respuesta adaptativa más del repertorio natural de la persona. Goleman y Cherniss (1998) están de acuerdo cuando inciden en que el aprendizaje social y emocional es diferente al cognitivo y técnico y por eso requiere un enfoque de entrenamiento y desarrollo distinto. La importancia de encontrar la manera de desarrollar este tipo de competencias nos exige descubrir otro tipo de metodología.

Vallés y Vallés (2003) apuntan en la misma línea cuando se refieren a que la formación en competencias socio-emocionales debería estar inserta en las distintas áreas curriculares, no en cuanto a su enseñanza/aprendizaje como contenido, sino como estilo educativo del docente que debe transmitir modelos emocionales adecuados. 
Nuestra propuesta de aplicación seria introducir la educación de estas competencias como materia transversal en al universidad, para ello también se debe contemplar una formación del profesorado universitario. De acuerdo con las conclusiones aportadas por diferentes estudios (Fernández Berrocal, Extremera y Palomera, 2008; Cabello, Ruiz-Aranda, Fernández-Berrocal 2010), los profesores deben recibir una formación que les permita desarrollar sus capacidades emocionales y sociales para que dicha formación no solo recaiga sobre ellos, sino también sobre su práctica docente. El EEES debe entenderse como una oportunidad para los alumnos, pero también para los profesores quienes a través de esta reforma pueden cuestionar y mejorar su práctica docente.

\section{Bibliografía}

Alberici, A., y Serreri, P. (2005). Competencias y formación en la edad adulta. Balance de competencias. Barcelona, Alertes.

Ashkanasy, N. M., y Daus, C. S. (2005) Rumors of the death of emotional intelligence in organizational behaivor are vastly exaggerated. Journal of Organizational Behavior, 26,441-452.

Austin, E. J., Saklofske, D. H., y Egan, V. (2005). Personality, well-being and health correlates of trait emotional intelligence. Personality and Individual Differences, 38(3), 547-558.

Baños, J. E., y Pérez, J. (2005). Cómo fomentar las competencias transversales en los estudios de Ciencias de la Salud: una propuesta de actividades. Educación Médica, 8(4), 216-225.

Bar-On, R. (1997). The Bar-On emotional quotient inventory (EQ-i): A test of emotional intelligence. Toronto, Canada: Multi-Health Systems.

Bisquerra, R. (2002). La práctica de la orientación y la tutoría. Barcelona, Praxis.

Cabello, R., Ruiz-Aranda, D., y Fernández-Berrocal, P. (2010). Docentes emocionalmente inteligentes. REIFOP, 13(1), 41-49.

Castejón, J. L., Cantero, M. P., y Pérez, N. (2008) Diferencias en el perfil de competencias socioemocionales en estudiantes universitarios de diferentes ámbitos científicos. Revista Electrónica de Investigación Psicoeducativa.15, 6(2) 339-362.

Cherniss, C. (2000) Social and emotional competence in the workplace. En R. Bar-On; J.D. Parker (eds.). The Handbook of Emotional Intelligence. San Francisco: Jossey-Bass.

Damasio, A. (2010).Y el cerebro creó al hombre. Barcelona Ediciones Destino.

Davies, M., Stankov, L., y Roberts, R. D. (1998). Emotional intelligence: in search of an elusive construct. Journal of Personality and Social Psychology, 75, 989- 1015.

Dawda, D., y Hart, S. D. (2000). Assessing emotional intelligence: reliability and validity of the Bar-On Emotional Quotient Inventory (EQ-I). Journal of Personality and Individual Differences, 28, 797-812.

Duncan, G. J., Dowsett, C. J., Claessens, A., Magnuson, K., Huston, A. C., Klebanov, P., et al. (2007). School readiness and later achievement. Developmental Psychology, 43, 1428-1446.

Durán-Aponte, E., y Durán-García, M. (2012) Competencias sociales y las prácticas profesionales. Vivencias y demandas para la formación universitaria actual. Cultura y Educación, 24(1) 61-76

Durlak, J. A., Weissberg, R. P., Dymnicki, A. B., Taylor, R. D., y Schellinger, K. B. (2011). The impact of enhancing students' social and emotional learning: A meta-analysis of school-based universal interventions. Child Development, 82, 405-432.

Fernández-Berrocal, P., Extremera, N.,y Palomera, R. (2008). "Emotional Intelligence as a crucial mental ability on educational context”. En A. Valle, y J. C. Nuñez (eds.), Handbook of Instructional Resources and their applications in the classroom. New York: Nova, 67-88.

Fernández-Berrocal, P. y Ruiz, D. (2008) La inteligencia emocional en la educación. Revista electrónica de Investigación Psicoeducativa. 15, 6(2) pp:421-436. 
Goleman, D., (1995). Inteligencia emocional. Barcelona, Kairós

Goleman, D. (1998). Working with Emotional intelligence. Londres, Bloomsburry Publishing Plc.

Goleman, D., Boyatzis, R., y McKee, A. (2002) El líder resonante crea más. Barcelona, Plaza y Janés.

Cherniss,C.,Goleman, D., Emmerling, R. J., Cowan, K., y Adler, M. (1998) (1998) Bringing emotional intelligence to the workplace. New Brunswick. NJ: Consortium for Research on Emotional Intelligence in Organizations, Rutgers University.

González, J. y Wagenaar, R. (2003). Tuning educational structures in europe. Final Report. Phase One. Bilbao: Universidad de Deusto.

Immordino-Yang, M. H., Damasio, A. (2006). We feel, therefore we learn: The relevance of affective and social neuroscience to education. Mind, Brain and Education, 1(1), 3-10.

Ley Orgánica 2/2006 de 3 de mayo de Educación. Oficial del Estado, núm. 106 de 4 de mayo de 2006, páginas 17158 a 17207.

Lopes, P. N., Côté, S., y Salovey, P. (2006). An ability model of emotional intelligence: Implications for assessment and training. In V. U. Druskat, F. Sala y G. Mount.

Mayer, J. D., Salovey, P. y Caruso, D. R. (2000). Models of emotional intelligence. In R. J. Sternberg (Ed.), Handbook of intelligence pp.396-420. Cambridge, UK: Cambridge University Press.

Neubauer, A. C. y Freudenthaler, H. H. (2005). Emotional intelligence: The convergent and discriminant validities of intra- and interpersonal emotional abilities. Personality and Individual Differences, 39, 569-579.

Palací, F. J. y Moriano, J. A. (coords.) (2003). El nuevo mercado laboral: estrategias de inserción y desarrollo profesional. Madrid: UNED.

Palací, F. J. y Topa, G. (coords.) (2002). La persona en la empresa: iniciativas de integración y desarrollo. Madrid: UNED.

Palomera, R. Fernández-Berrocal, P., y Brackett, M.A. (2008). La inteligencia emocional como una competencia básica en la formación inicial de los docentes: algunas evidencias. Revista electrónica de investigación Psicoeducativa, 15 6(2) 437-454

Pérez Escoda, N., Filella, G. y Soldevila, A., (2010). Competencias emocional y habilidades en estudiantes universitarios. Revista de Motivación y Emoción 2012, 1, 22-30.

Petrides, K. V. y Furnham, A. (2003). Trait of emotional intelligence: Behavioral validation in two studies of emotion recognition and reactivity to mood induction. European Journal of Personality, 17, 39-57.

Repetto, E., Ballesteros, B. y Malik, B. (2000). Tareas y formación de los orientadores en la Unión Europea. Madrid. UNED

Repetto, E., y Pérez-González, J.C. (2007). Formación en competencias socioemocionales a través de las prácticas en empresas. Revista Europea de Formación Profesional, 40, 92-112.

Roe, R.A. (2002). Competences: A key towards the integration of theory and practice in work psychology. Gedrag en Organisatie, 15, 203-224.

Sala, J. (2002). Ideas previas sobre la docencia y competencias emocionales en estudiantes de ciencias de la educación. Revista Española de Pedagogía. Año LX, 223, 543-558.

Vallés, A. y Vallés, C. (2003). Psicopedagogia de la Inteligencia Emocional. Valencia. Promolibro. 


\section{Autores}

Marta Fortes Vilaltella

Profesora Asociada a tiempo parcial a las áreas de MIDE y DOE. Ha impartido las materias de; Sociedad, Familia y Escuela II, Observación Sistemática y análisis de contextos II, Procesos y Contextos Educativos III, Dificultades de Aprendizaje, en los Grados de Educación Primaria y Educación Infantil. Profesora en el máster de Educación Secundaría y Investigación en Educación. Participa en un proyecto de innovación docente del Departamento. Miembro del ICE de la UdL

\section{Xavier Oriol Granado}

Personal Investigador en Formación del Departamento de Pedagogía y Psicología. Imparte la asignatura de las Áreas de MIDE y Psicología Social en la asignatura: Sociedad, Familia y Escuela

Gemma Filella Guiu

Profesora Titular de Universidad (TUC) del Departamento de Pedagogía y Psicología, Área de MIDE. Coordinadora del Máster de Inteligencia emocional en las Organizaciones. Coordinadora del grupo ICE de educación emocional de la UdL. Su perfil investigador se centra en la orientación psicopedagógica en general y más en concreto en la educación emocional

Isabel del Arco

Profesora Titular de Universidad (TUC) del Departamento de Pedagogía y Psicología, Área de Didáctica y Organización de Centros. Imparte clases en el Grado de Educación Primaria: Procesos y Contextos Educativos, Diseño Curricular, Programación y elaboración de materiales en el máster Universitario de Enseñanza de Catalán y Castellano para inmigrantes. Fundamentos en Investigación Educativa en el máster de Investigación Educativa. También participa como docente en propuestas formativas de postgrado y máster en países Iberoamericanos. Participa en diversas propuestas de innovación docente como: Modelo de Formación en alternancia, entre muchos otros

Anna Soldevila Benet

Profesora del área de Didáctica y Organización Escolar. Imparte clases en los Grados de Educación Social y Trabajo Social: Educación y Comunicación, Conceptos Básicos de Educación, Estrategias de Acción Socioeducativas I. Coordinadora del postgrado en Educación Emocional y Bienestar. Miembro del Grupo de Investigación en Orientación Psicopedagógica (GROP), Miembro del grupo ICE de la UdL 